\title{
Optimizing the Choice of Spin-Squeezed States for Detecting and Characterizing Quantum Processes
}

\author{
Lee A. Rozema, ${ }^{1}$ Dylan H. Mahler, ${ }^{1}$ Robin Blume-Kohout, ${ }^{2}$ and Aephraim M. Steinberg ${ }^{1,3}$ \\ ${ }^{1}$ Department of Physics, Centre for Quantum Information and Quantum Control, \\ and Institute for Optical Sciences, University of Toronto, 60 St. George Street, \\ Toronto, Ontario, Canada M5S $1 A 7$ \\ ${ }^{2}$ Sandia National Laboratories, Computer Science Research Institute (CSRI), \\ Albuquerque, New Mexico 87123, USA \\ ${ }^{3}$ Canadian Institute for Advanced Research, Toronto, Ontario M5G 1Z8, Canada
}

(Received 22 May 2014; published 7 November 2014)

\begin{abstract}
Quantum metrology uses quantum states with no classical counterpart to measure a physical quantity with extraordinary sensitivity or precision. Most such schemes characterize a dynamical process by probing it with a specially designed quantum state. The success of such a scheme usually relies on the process belonging to a particular one-parameter family. If this assumption is violated, or if the goal is to measure more than one parameter, a different quantum state may perform better. In the most extreme case, we know nothing about the process and wish to learn everything. This requires quantum process tomography, which demands an informationally complete set of probe states. It is very convenient if this set is group covarianti.e., each element is generated by applying an element of the quantum system's natural symmetry group to a single fixed fiducial state. In this paper, we consider metrology with 2-photon ("biphoton") states and report experimental studies of different states' sensitivity to small, unknown collective $S U(2)$ rotations ["SU(2) jitter"]. Maximally entangled $N 00 N$ states are the most sensitive detectors of such a rotation, yet they are also among the worst at fully characterizing an a priori unknown process. We identify (and confirm experimentally) the best $S U(2)$-covariant set for process tomography; these states are all less entangled than the $N 00 N$ state, and are characterized by the fact that they form a 2-design.
\end{abstract}

DOI: 10.1103/PhysRevX.4.041025

\section{INTRODUCTION}

The goal of quantum metrology is to measure or detect physical phenomena with surprising precision by exploiting quantum resources. Often, this means using entangled states to achieve greater resolution or sensitivity. For example, squeezed light [1] and NOON states [2-4] have been used in interferometers to achieve higher precision in single parameter estimation. $N 00 N$ states are maximally sensitive to small $U(1)$ phase shifts [5], but they are fragile. Other parameters might be best detected or estimated by a different optimal state [6,7], and for estimating even a simple three-parameter $S U(2)$ process, the optimal state is unknown [8]. At the opposite extreme of metrology is quantum process tomography (QPT) $[9,10]$. Here, the goal is to learn every parameter of an unknown process. QPT requires a diverse set of probe states, and the overall accuracy of estimation depends on the properties of the entire set. For process tomography on a single quantum

Published by the American Physical Society under the terms of the Creative Commons Attribution 3.0 License. Further distribution of this work must maintain attribution to the author(s) and the published article's title, journal citation, and DOI.
Subject Areas: Quantum Physics, Quantum Information

optical mode, Lobino et al. [11] showed that it is sufficient to (1) prepare a single Glauber coherent state and (2) displace it by a variety of phase space translations. This approach, in which a single "fiducial" state is multiplied into a complete set of probe states by easily implemented group transformations, has the great merit of experimental ease. But while sufficiently large coherent-state ensembles are sufficient for process tomography, they are not efficient. Coherent states are very "classical" [12], and provide exponentially little information about parameters of some quantum processes, motivating a search for a set of states for tomography that provide equal information about all possible processes (see Ref. [13] for a precise statement of this problem).

In this paper, we examine a closely related question for 2-photon polarization ("biphoton") states. Like an optical mode, this system admits (spin-)coherent states (as well as others). The corresponding symmetry group, $S U(2)$, is transitive on the set of coherent states; i.e., a spin-coherent state can be transformed into any other spin-coherent state by applying a polarization $[S U(2)]$ rotation. We prepare a wide range of probe states and quantify their performance at two opposite extremes of the metrology spectrum: (1) their ability to detect random $S U(2)$ phase shifts, and (2) their ability to characterize an unknown process, 
when displaced by a variety of $S U(2)$ rotations and used for QPT. Remarkably, the most sensitive detector states ( $N 00 \mathrm{~N}$ states) are also among the least effective for QPT. The optimal $S U(2)$-covariant set [i.e., a set generated by applying $\mathrm{SU}(2)$ operations to a fiducial state] for QPT is generated by a state that is neither spin coherent nor $N 00 N$, but outperforms both of them. When displaced by uniformly random $S U(2)$ operations, it generates a 2 -design $[14,15]$, confirming the theoretical prediction that 2-designs should be optimal for process tomography.

We focus on a particular, important family of processes that we call $S U(2)$ jitter. In $S U(2)$ jitter, an $N$-photon state experiences a small random collective $S U(2)$ rotation, whose magnitude is Gaussian distributed. Detecting and characterizing $S U(2)$ jitter is important because it is a common model for decoherence [16], the primary enemy of quantum information and computation [17]. Noiseless, subsystems [18,19] were designed against this noise model.

\section{EXPERIMENTAL METHODS AND PRELIMINARY VALIDATIONS}

A biphoton is a system of two photons in the same spatial and temporal modes [20,21]. Basis states can be described by specifying the number of photons polarized horizontally $(H)$ and vertically $(V)$; e.g.,

$$
C_{0}|2,0\rangle_{H, V}+C_{1}|1,1\rangle_{H, V}+C_{2}|0,2\rangle_{H, V} .
$$

A single-photon polarization state is a two-level system and it is isomorphic to a spin- $1 / 2$ particle. We can define a single horizontally polarized photon to be a spin-up spin$1 / 2$ particle and a vertically polarized photon to be a spindown spin-1/2 particle. Similarly, the polarization state of a biphoton is isomorphic to a spin-1 particle [22]. Hence, we can write a general biphoton state in the angular-momentum basis $\{|J=1, m=+1\rangle,|J=1, m=0\rangle,|J=1, m=-1\rangle\}$ as

$$
C_{0}|1,+1\rangle+C_{1}|1,0\rangle+C_{2}|1,-1\rangle,
$$

where the equivalence between the two biphoton bases can be seen by comparing Eqs. (1) and (2). A collective polarization rotation corresponds to an $S U(2)$ rotation of the effective spin-1 particle about some axis $\vec{r}$ by an angle $\theta$.

Two photons polarized in the same direction form a spincoherent state, and these spin-coherent states are analogous to the Glauber coherent states of an optical mode [23]. Just as Ref. [11] used displaced Glauber coherent states as input states for QPT, a set of at least nine distinct spin-coherent states can form a complete probe set for QPT, and can be generated by applying various $S U(2)$ rotations to a single fiducial spin-coherent state. Here, we generalize this procedure in a simple way: we prepare a fiducial state that is not necessarily a spin-coherent state and generate candidate probe sets for QPT by applying ten distinct
$S U$ (2) rotations to it. (We prepare ten fiducial states instead of nine because it is experimentally convenient and provides a small amount of useful redundancy.) Our fiducial states take the form

$$
\left|\psi_{x}\right\rangle=\sqrt{x}|2,0\rangle_{H, V}+\sqrt{1-x}|0,2\rangle_{H, V}
$$

and are prepared using the apparatus sketched in Fig. 1(a) and the methods described in Refs. [24-29].

In brief, we prepare our biphoton states by combining vertically polarized laser light with horizontally polarized collinear down-converted light into the same spatial mode by overlapping them at a polarizing beam splitter. We then postselect on 2-photon events in this mode. Since down conversion only produces photons in pairs, we can only ever detect two horizontally polarized down-converted photons or two vertically polarized laser photons. If the photons from both sources are indistinguishable, this process will result in the superposition state of Eq. (3). To set $x$, we tune the amplitude of the laser light while keeping the amplitude of the down-converted light fixed. We actively stabilize the phase between the laser light and the down-converted light, allowing for data runs lasting several hours [29]. The class of biphoton states that we can prepare [Eq. (3)] includes spin-coherent states $(x=0,1)$ and the two-photon $N 00 N$ state $\left(x=\frac{1}{2}\right)$. In fact, any biphoton state can be prepared by choosing some value of $x$ and then applying some $S U(2)$ rotation using wave plates. [ $N$-photon states have $N-1$ parameters that are $S U$ (2) invariant; for the biphoton, $x$ is the only parameter.]

The horizontally and vertically polarized photons are not perfectly mode matched when generated. We remedy this by passing them through a $3 \mathrm{~nm}$ filter and coupling them into a single-mode fiber, which discards any photons not in the desired mode. This procedure results in near-perfect biphotons, as quantified by the (very small, $<2 \%$ ) amount of population in the antisymmetric subspace. We characterize our state preparation by doing quantum state tomography using the apparatus of Fig 1(c) as described in Refs. [21,22]. To visualize quantum polarization states on the Poincaré sphere, we must use a quasiprobability distribution because arbitrary $N$-photon polarization states cannot be represented as a point on the Poincaré sphere (as can be done with classical light). We use Wigner distributions as defined in Ref. [30]. Our resulting experimentally measured biphoton states, after numerically filtering out the antisymmetric subspace, are depicted in the first column of Fig. 2 as Wigner distributions plotted on the Poincare sphere [31]. As $x$ is increased, the states become more "nonclassical," with the most nonclassical state being the N00N state with $x=0.5$ [see column 1 of Fig. 2(c)]. This procedure lets us prepare any desired state with fidelity $\geq 93 \%$.

The process we study, depolarization due to fluctuating $S U(2)$ rotations, manifests itself in many experimental 


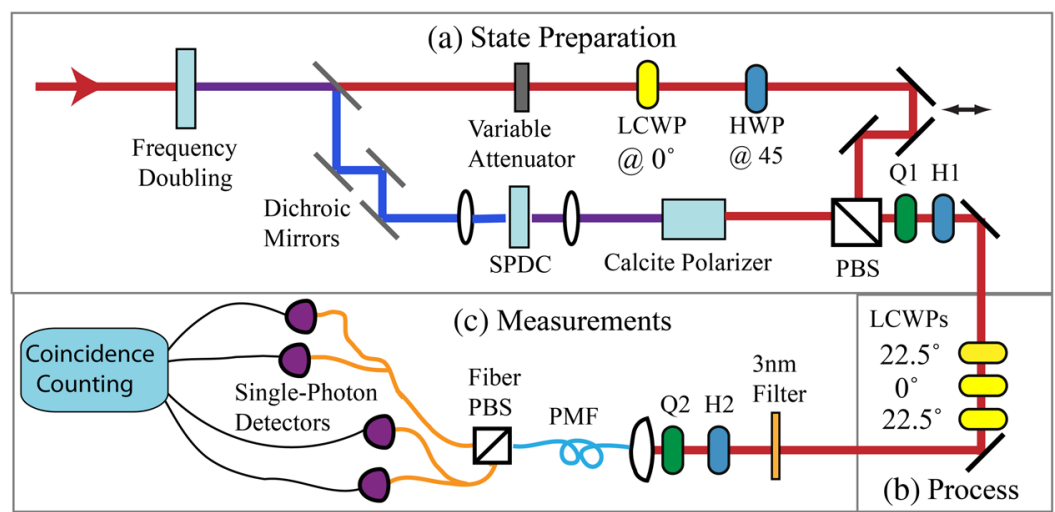

(d) Input States

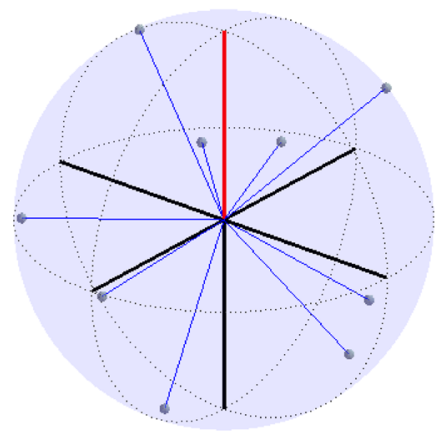

FIG. 1. Schematic diagram of the experimental apparatus used to generate and measure different biphoton states. (a) State preparation. We can prepare any biphoton state in two steps. First, we prepare a state of the form $\sqrt{x}|2,0\rangle_{H, V}+e^{i \phi} \sqrt{1-x}|0,2\rangle_{H, V}$ by using a polarizing beam splitter (PBS) to combine a vertically polarized weak coherent state with the output of horizontally polarized type-I collinear spontaneous parametric down-conversion (SPDC). The amplitude $x$ is set by attenuating the laser intensity relative to the down-converted intensity. The angle $\phi$ is set by the relative phase between the two paths, which we control using a liquid-crystal wave plate (LCWP). Then, we apply any desired polarization rotation in $S U(2)$, using quarter-wave and half-wave plates, to produce any desired biphoton state. (b) Process. Three LCWPs, oriented as shown, are used to perform arbitrary polarization rotations. To implement depolarization, the retardances of the LCWPs are made to fluctuate during each measurement. (c) State measurement. The biphoton states pass through wave plates, are coupled into a polarization-maintaining fiber (PMF), and are sent to a polarizing beam splitter. The output of each port of the beam splitter is probabilistically split, using 50:50 fiber beam splitters, and sent to single-photon counting modules. (d) Input states. A graphical representation of the set of states used for process tomography. A fiducial state is prepared and rotated to nine other states. These states (including the unrotated fiducial state) make up the set of ten input states used for process tomography. The fiducial state is represented by the red line, and it is rotated to each of the nine other points on the sphere. The rotations are chosen to be (approximately) uniformly distributed on the surface of the sphere.

systems-e.g., a spin in a fluctuating magnetic field, or a polarization state propagating through an optical fiber that thermally fluctuates with time. In both cases, the state (the spin state or the polarization state) couples to an external system (a time-varying field or a time-varying birefringence), causing depolarization. For example, a spin is

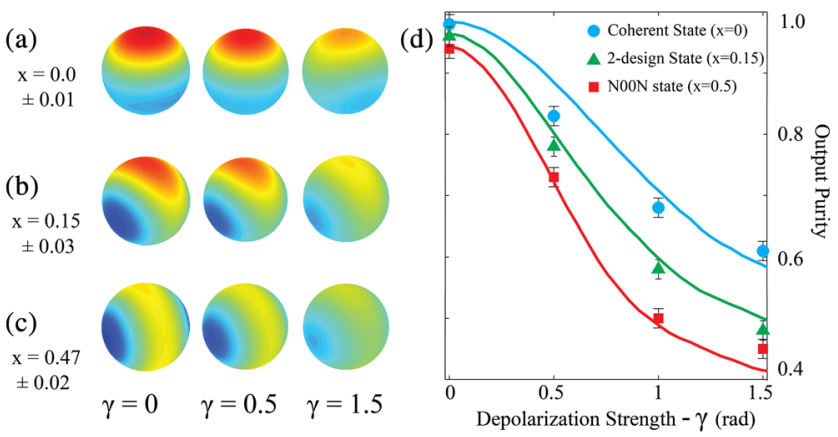

FIG. 2. Typical results of state tomography and the effect of $S U$ (2)-jitter depolarization. Panels (a)-(c) show experimentally reconstructed Wigner functions (plotted on the Poincaré sphere) for three different states after they have been decohered by three different amounts of $S U(2)$ jitter. (a) Spin-coherent states with $x=0$, (b) "2-design" states with $x=0.15$, and (c) nearly $N 00 N$ states with $x=0.47$. Each row shows the effect of applying depolarization with strength [see Eq. (4)] $\gamma=0,0.5,1.5$. In (d), we plot the purity of the same reconstructed states shown in (a)-(c). The solid lines are the theoretical predictions given by simulations of the process [Eq. (4)]. coupled to a magnetic field via the Hamiltonian $\hat{H}=-\mu \vec{J} \cdot \vec{B}$, where $\vec{B}=B_{o} \vec{r}$ (the direction of the magnetic field is $\vec{r}$ and the strength of the magnetic field is $B_{o}$ ). This has the effect of rotating the spin about an axis $\vec{r}$ by an angle $\theta=\mu B_{o}$, as described by the unitary operator $\hat{U}=e^{-i \theta(\vec{r} \cdot \vec{J} / \hbar)}$. If $\vec{B}$ changes with time, then the output state of this process can be described by properly averaging over $\vec{r}$ and $\theta$ [32]. (Polarization states are similarly rotated by a birefringent refractive index, where $\theta$ is related to the strength of the birefringence and $\vec{r}$ is related to the orientation of the optic axis.) In $S U(2)$ jitter, we consider isotropic depolarization (by which we mean that there is no preferred rotational axis) and we assume that $\vec{r}$ and $\theta$ are uncorrelated. In this case, the quantum process is

$$
D_{\gamma}[|\psi\rangle\langle\psi|]=\int d \vec{r} \int d \theta P(\theta) e^{-i \theta(\vec{r} \cdot \vec{J} / \hbar)}|\psi\rangle\langle\psi| e^{+i \theta(\vec{r} \cdot \vec{J} / \hbar)},
$$

where the rotation axis $\vec{r}$ is uniformly random and the angle $\theta$ has a distribution, $P(\theta)$, which we take to be Gaussian:

$$
P(\theta) \propto e^{-\theta^{2} / 2 \gamma^{2}}
$$

The overall strength of the depolarization process is quantified by $\gamma$, the width of the distribution of $\theta$. 
We implement the depolarization process described by Eq. (4) using three liquid-crystal wave plates (LCWPs) as shown in Fig. 1(b). Each LCWP applies an adjustable polarization rotation, and the rotation angle can be changed very rapidly. This allows us to easily apply 50 different (randomly selected) rotations to implement a single process, closely approximating the ideal process of Eq. (4) as a statistical mixture of these 50 random unitary processes:

$$
\tilde{D}_{\gamma}[|\psi\rangle\langle\psi|]=\sum_{k=1}^{50} e^{-i \theta_{k}\left(\vec{r}_{k} \cdot \vec{J} / \hbar\right)}|\psi\rangle\langle\psi| e^{+i \theta_{k}\left(\vec{r}_{k} \cdot \vec{J} / \hbar\right)}
$$

Using this implementation, we can apply precisely calibrated $S U(2)$ jitter. We verify both our state preparation and our implementation of depolarization by performing state tomography on $\rho=\tilde{D}_{\gamma}\left[\left|\psi_{x}\right\rangle\left\langle\psi_{x}\right|\right]$ for several values of $x$ and $\gamma$. Figures 2(a)-2(c) show the Wigner functions of the resulting reconstructed states. Plots in column 1 are for undecohered states, while columns 2 and 3 show the effects of $S U(2)$ jitter with strengths of $\gamma=0.5 \mathrm{rad}$ and $\gamma=1.5 \mathrm{rad}$, respectively. Increasing $\gamma$ blurs the Wigner function. This is captured quantitatively by the state's purity, a reasonable proxy for the amount of depolarization suffered. Figure 2(d) plots the output purity (computed from the tomographic estimate) versus $\gamma$, and compares it to the prediction of numerical simulations of $\tilde{D}_{\gamma}$ (solid lines), for three different input states with $x=0$ (blue), $x=0.15$ (green), and $x=0.47$ (red). The only inputs to our simulation are the experimentally measured purities of the input states when $\gamma=0$, which in a perfect experiment would be 1 , but are slightly degraded by experimental noise. We observe excellent agreement between simulation and experiment, confirming that our process performs as expected. In particular, the $N 00 N$ state loses purity more rapidly than any other state (as $\gamma$ is increased), indicating that $N 00 N$ states are indeed the most fragile (and thus potentially sensitive) to $S U(2)$ jitter. The fragility of $N 00 \mathrm{~N}$ states to a similar model of $S U(2)$ depolarization was also pointed out in Ref. [16]. In the next section, we discuss an experiment exploiting this fragility to detect depolarization.

\section{DETECTING DEPOLARIZATION}

We examine the effectiveness of different probe states in detecting $S U(2)$ jitter. This corresponds to distinguishing between two processes: 11 (no depolarization) or $D_{\gamma}$ [SU(2) jitter]. Acting on the probe state $\left|\psi_{x}\right\rangle\left\langle\psi_{x}\right|$, these alternatives produce either $\left|\psi_{x}\right\rangle\left\langle\psi_{x}\right|$ or $\rho_{D}=D_{\gamma}\left[\left|\psi_{x}\right\rangle\left\langle\psi_{x}\right|\right]$, and to distinguish these alternatives, we perform a positive operator value measure (a generalized measurement [32]) with two outcomes:

$$
\mathcal{M}=\left\{\left|\psi_{x}\right\rangle\left\langle\psi_{x}|, 11-| \psi_{x}\right\rangle\left\langle\psi_{x}\right|\right\}
$$

In simple terms, we are checking to see whether the probe state changes at all. If done perfectly, this protocol has onesided error; it may fail to detect $D$, but will never detect it in error.

To implement this measurement experimentally, we recall that if $\left|\psi_{x}\right\rangle$ is a spin-coherent state, then it can be written as $\hat{U}|2,0\rangle_{H, V}$ for some $\hat{U} \in S U(2)$. We can implement $\hat{U}^{\dagger}$ using the quarter- and half-wave plates (HWP) labeled Q2 and H2 in Fig. 1(c), and after performing this inverse rotation on the output state, detection of two photons at the $H$ port of the polarizing beam splitter (PBS) corresponds to the $\left|\psi_{x}\right\rangle\left\langle\psi_{x}\right|$ outcome of $\mathcal{M}$. Similarly, if $\left|\psi_{x}\right\rangle$ is a $N 00 N$ state, it can be written as

$$
\begin{aligned}
\left|\psi_{x}\right\rangle & =\frac{1}{\sqrt{2}}\left(|2,0\rangle_{H, V}+|0,2\rangle_{H, V}\right) \\
& =U|1,1\rangle_{H, V},
\end{aligned}
$$

where $U^{\dagger}$ can be implemented by a HWP at $22.5^{\circ}$. Thus, after performing this inverse rotation on the output state, detection of two coincident photons at the $H$ and $V$ ports of the PBS corresponds to the $\left|\psi_{x}\right\rangle\left\langle\psi_{x}\right|$ outcome of $\mathcal{M}$.

In our experiment, for states with $x \neq 0$ or $x \neq 0.5$, we estimate the value $\left\langle\mid \psi_{x}\right\rangle\left\langle\psi_{x} \mid\right\rangle$ indirectly from two measurements. In general, the density matrix describing the system at the output is

$$
\rho=\left(\begin{array}{lll}
a & f & d \\
f^{*} & b & g \\
d^{*} & g^{*} & c
\end{array}\right),
$$

where $\rho$ is written in the same basis as the state in Eq. (3). If the process is pure $S U(2)$ depolarization, and the input state is given by Eq. (3), then $d$ will be real. (We check that this is the case by performing quantum state tomography on $\rho$ for several depolarization strengths.) In this case, the expectation value of the projection onto $\left|\psi_{x}\right\rangle$ is

$$
\begin{aligned}
\left\langle\mid \psi_{x}\right\rangle\left\langle\psi_{x} \mid\right\rangle & =\operatorname{Tr}\left(\left|\psi_{x}\right\rangle\left\langle\psi_{x}\right| \rho\right) \\
& =a x+c(1-x)+2 d \sqrt{x(1-x)} .
\end{aligned}
$$

So we can estimate $\left\langle\mid \psi_{x}\right\rangle\left\langle\psi_{x} \mid\right\rangle$, for any value of $x$, by measuring $a, c$, and $d$. If $\rho$ is sent directly to a PBS, both photons will be transmitted with probability $a$, both will be reflected with probability $c$, and one will exit each port with probability $b$. All of these probabilities can readily be measured via coincident detection between different combinations of the four detectors in Fig 1(c). To measure $d$, a half-wave plate at $22.5^{\circ}$ is inserted before the PBS. Now, one photon will exit each port of the PBS with probability

$$
P_{H V}=\frac{1}{2}-d-\frac{b}{2}
$$


Since $b$ is already known, measuring $P_{H V}$ gives us an estimate of $d$, which gives us enough information to reconstruct $\left\langle\mid \psi_{x}\right\rangle\left\langle\psi_{x} \mid\right\rangle$ for any value of $x$. While our "two-step" method works, it is possible to directly perform this projection for any biphoton state. One way to do this is by time reversing the state preparation techniques of Refs. [3,25,33], where it is shown how arbitrary $N$-photon polarization states (occupying a single spatial mode) can be built up by combining $N$ photons one at a time into the same mode. The polarization of each individual photon sets the $N$-photon polarization state. The time reverse of this process would be to split a single-mode $N$-photon polarization state into $N$ separate spatial modes (probabilistically) and to detect a single photon with a specific polarization in each mode. Thus, this would correspond to projecting onto a state which is defined by the $N$ different polarization measurements.

We note in passing that this protocol is reminiscent of atomic interferometry. There, too, a probe state is prepared and then measured later. The probability (and statistics) of the results typically oscillates over time, because different atomic states have different energies and accumulate quantum phases that beat against one another. Depolarization makes these oscillations decay and eventually disappear, and this decay is often used to estimate the depolarization strength in the system. Thus, in both interferometry and our experiment, greater fragility to depolarization enables detection of weaker depolarization.

We create a range of probe states parametrized by $x \in\left[0, \frac{1}{2}\right]$, varying from a spin-coherent state at $x=0$ to a $N 00 N$ state at $x=\frac{1}{2}$ [Fig. 1(a)], subject them to depolarization [Fig. 1(b)], and perform the measurement $\mathcal{M}$ given in Eq. (7) [Fig. 1(c)].

Figure 3(a) shows the experimentally observed probability of failing to detect $S U(2)$ jitter as a function of the jitter's strength $(\gamma)$, for three representative probe states: a $N 00 N$ state (red), a spin-coherent state (blue), and an intermediate state with $x=0.15$ (green). The experimental results are in good agreement with the simple theoretical prediction (solid lines) given by

$$
\begin{aligned}
P(\gamma) & =\left\langle\psi_{x}\left|D\left[\left|\psi_{x}\right\rangle\left\langle\psi_{x}\right|\right]\right| \psi_{x}\right\rangle \\
& =A_{x} e^{-2 \gamma^{2}}+B_{x} e^{-\gamma^{2} / 2}+C_{x},
\end{aligned}
$$

where $A_{x}, B_{x}$, and $C_{x}$ are straightforward but unwieldy functions of $x$ [see Eq. (4) in Sect. A of the Supplemental Material for their form [34]]. We see that the $N 00 N$ state is consistently the best detector of $S U(2)$ jitter, and that detection probability for any fixed depolarization strength appears to increase (as expected) monotonically with $x$.

Metrology is also concerned with estimating (rather than just detecting) parameters of a process. In this case, that means estimating $\gamma$, and this requires repeating the experiment more than once, since a single experiment can at best

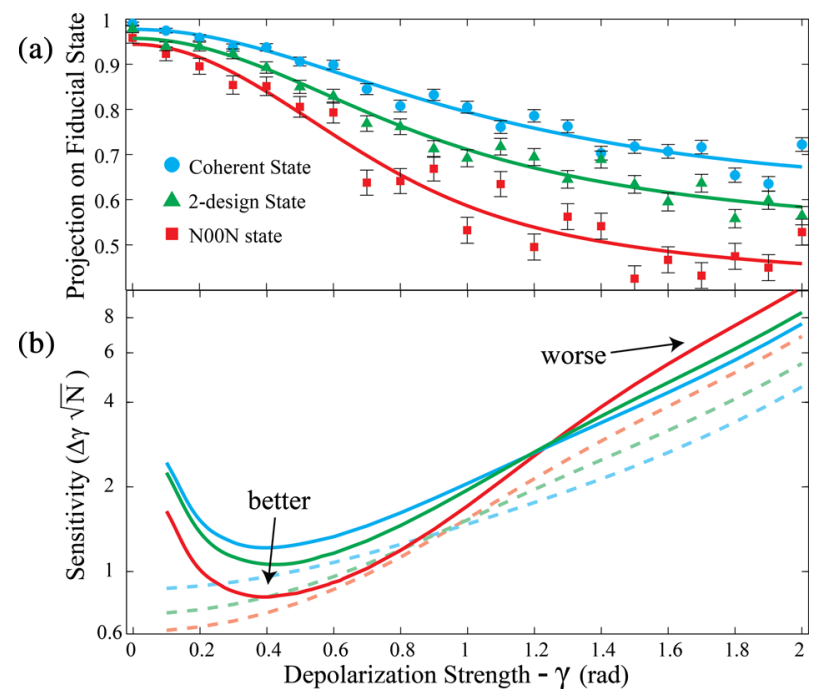

FIG. 3. Sensitivity of different states to depolarization. This figure shows two different measures of the probe state's ability to detect depolarization, for three different probe states, and compares theoretical predictions to experimental data. Panel (a) shows the probability that depolarization is not detected, which is simply the probability of finding the system in the same state in which it was prepared despite depolarization having happened. The theory (solid line) is simply the projection $\left\langle\psi_{x}|\rho| \psi_{x}\right\rangle$, where $|\psi\rangle_{x}$ is the probe state and $\rho$ is the decohered probe state. Experimental data points are empirical probabilities of nondetection. Panel (b) shows the sensitivity [Eq. (12)] scaled by $\sqrt{N}$ (where $N$ is the number of 2-photon counts detected at each point) of each state to small changes in depolarization; the solid lines are calculated from the slopes of the fit to the detection data shown in (a), and the dashed lines are calculated for ideal input states.

detect that $\gamma>0$. We can then estimate the probability plotted in Fig. 3(a), e.g., as $\hat{P}=n / N$, where the experiment is repeated $N$ times and depolarization is detected in $n$ of them. Armed with our knowledge of the initial state and our estimate $\hat{P}$ of the nondetection probability, we then estimate $\gamma$ [e.g., by simply inverting the appropriate theoretical curve shown in Fig. 3(a)]. Of course, our estimate $(\hat{\gamma})$ has some uncertainty:

$$
\gamma=\hat{\gamma} \pm \Delta \gamma
$$

$\Delta \gamma$ is the smallest change in $\gamma$ that can be detected with reasonable probability. We refer to it as sensitivity (although it should be noted that smaller $\Delta \gamma$ implies greater sensitivity), and it is given by [35]

$$
\Delta \gamma=\frac{\Delta P(\gamma)}{\frac{d P(\gamma)}{d \gamma}},
$$

where $\Delta P(\gamma)$ is the standard deviation of the estimated nondetection probability $\hat{P}$. Since our detection protocol is a Bernoulli (coin-flip) process, $\Delta P(\gamma)=\sqrt{P(\gamma)(1-P(\gamma)) / N}$. 
Both $P(\gamma)$ and $d P(\gamma) / d \gamma$ depend only on $x$ (a property of the probe state) and can be computed from Eq. (11).

In Fig. 3(b), we show the dependence of sensitivity, scaled by $\sqrt{N}$ (where $N$ is the number of times the experiment is repeated) on $\gamma$ and the probe state. We scale the sensitivity by $\sqrt{N}$ so that it only depends on the input state and $\gamma$. Since sensitivity is not a directly observable quantity, we compare a pure theory prediction to an empirical fit. Dashed lines are pure theoretical predictions, in which both $P(\gamma)$ and $d P(\gamma) / d \gamma$ are calculated using Eq. (11) for ideal input states. The solid lines are empirical fits: both $P(\gamma)$ and $d P(\gamma) / d \gamma$ are calculated from the smooth empirical fits to data shown as solid lines in Fig. 3(a).

Whereas N00N states are always the best detectors of depolarization, we observe that they are only the best at measuring $\gamma$ for low values of $\gamma$. Around $\gamma \approx 1$, they become less sensitive than the other states. This is a direct consequence of their extreme fragility; since almost any amount of depolarization disturbs the $N 00 N$ state, it does not distinguish well between medium and strong depolarization. We also observe a significant discrepancy between the ideal sensitivity (dashed lines) and the observed value at small $\gamma$. This is because our input states are imperfect and not pure-even at $\gamma=0$, there is a small probability that we will (falsely) detect depolarization. Thus, $P(\gamma)$ is never 1 , and $\Delta P(\gamma) \nrightarrow 0$ as $\gamma \rightarrow 0$. Still, we find that $N 00 N$ states are clearly optimal for $\gamma \lesssim 0.9$, beating spin-coherent states by a factor of $1.44 \pm 0.05$, which agrees well with our theoretical prediction of $\sqrt{2}$ (see Sect. A of the Supplemental Material for derivation [34]).

\section{QUANTUM PROCESS TOMOGRAPHY}

Using the protocol above, we can detect depolarization, and we can even quantify its strength. But to do so reliably, we need to assume that the process is of a specific oneparameter form [pure $S U(2)$ jitter]. For example, a consistent and coherent $S U(2)$ rotation would violate this assumption, and might go entirely undetected or be incorrectly diagnosed as jitter (depending on the axis of the measurement and the nature of the probe state). Characterizing general depolarization processes, and correctly diagnosing what is happening, requires quantum process tomography $[9,10]$.

QPT requires not one, but an ensemble of input statesand preparing a suitable ensemble can be quite challenging. We avoid this complexity by using the ideas of Lobino et al. [11] and generating diverse input states by applying diverse simple transformations to a single fiducial state [see Fig. 1(d)]. Lobino et al. prepared optical coherent states by translating the $|0\rangle$ state. The equivalent protocol in our biphoton system is to prepare the $|2,0\rangle_{H, V}$ state and then generate an ensemble of spin-coherent states by performing different $S U(2)$ rotations on it. However, we go one step further and generalize this process by varying the fiducial state (parametrized, again, by $x \in[0,1]$ ). We study the dependence of process reconstruction fidelity on $x$, to determine (in particular) whether the $N 00 \mathrm{~N}$ states that best detect depolarization are also the best probe states to characterize it.

QPT reconstructs the entire process matrix (or superoperator) from the observed measurement statistics. This reconstruction, in essence, involves solving a set of linear equations described by a the Gram matrix $M$ of the input states. The reconstructed process is obtained by applying $M^{-1}$ to a vector of observed statistics. $M$ must obviously be full rank, but (moreover) it should not have any small eigenvalues. If $M$ has small eigenvalues, $M^{-1}$ will amplify small statistical fluctuations that result from finite sample size into large errors, and the reconstructed process will have low fidelity with the true process.

This property (the "tomographic power" of a set of probe states) is hard to quantify exactly, but it can be captured approximately by the determinant of the Gram matrix. This is zero if (and only if) the probe ensemble completely fails to probe at least one dimension of state space, and it achieves its maximum value if (and only if) the probe ensemble is a 2-design. Larger determinants are better, implying that the inversion will amplify errors less. In the inset of Fig. 4, we plot this determinant as a function of the fiducial state's $x$ value. For a $N 00 N$ state $(x=1 / 2)$, the determinant is zero. So, remarkably, the set of states generated by the $S U(2)$ orbit of a $N 00 N$ state is incomplete-it does not enable QPT at all, and is completely oblivious to at least one parameter of the process [the Gram matrix of the $S U(2)$-covariant set generated using a $N 00 N$ state is shown to be rank deficient in Sect. B of the Supplemental Material [34]]. At the opposite extreme $(x=0)$, spin-coherent states do generate a complete set, suitable for QPT, but, like the coherent states of Lobino et al., they are not optimal for the task. The determinant is small, indicating that at least one parameter of the process is poorly resolved. The maximum value of the determinant is achieved at an intermediate point, $x=1 / 2-(1 / 2 \sqrt{2}) \approx 0.15$, which generates a set of probe states that are neither $N 00 N$ nor spin coherent. We predicted that this set of states would enable optimally accurate QPT.

The $x \approx 0.15$ ensemble is special and unique in another way; it forms a 2-design (as shown in Sect. B of the Supplemental Material [34]). Informally, 2-designs are sets of quantum states whose projectors span the vector space of operators as uniformly as possible; more precisely, the ensemble's second moments are equal to those of the uniform Haar ensemble over pure states. Common examples of 2-designs include mutually unbiased bases [36] and symmetric informationally complete measurements [37,38], and there is strong theoretical [36] and experimental [39] evidence that 2-designs are optimal for state tomography. Our results here are the first experimental evidence that 2-designs are optimal for QPT (see theory in Refs. $[14,15]$ ). 


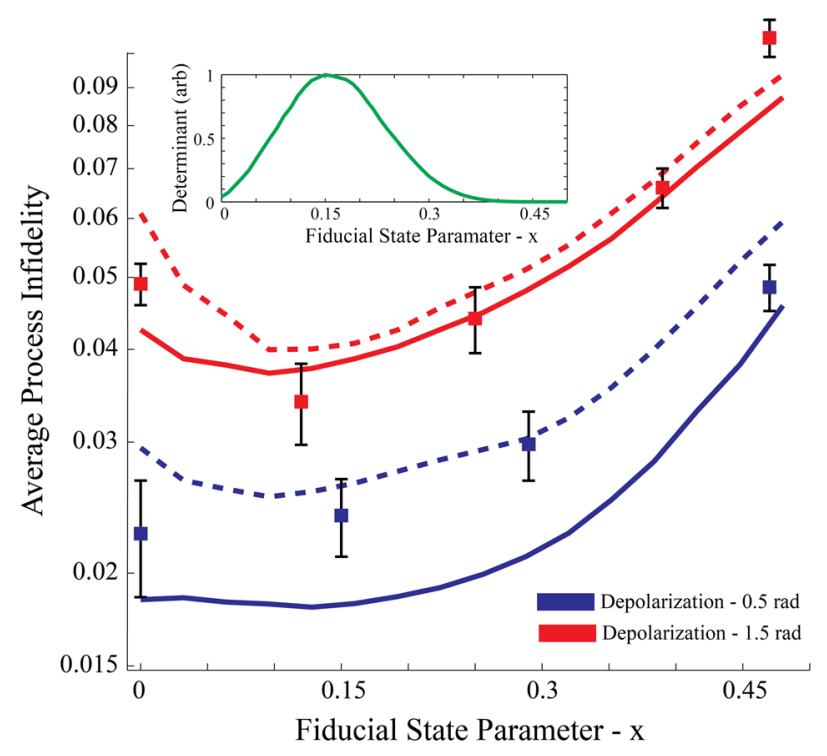

FIG. 4. Inaccuracy of process reconstruction. This figure shows the average process infidelity (API) [see Eq. (13) - smaller is better] between the true process and its tomographic reconstruction, and its dependence on the fiducial state used to generate the set of probe states for QPT. Solid lines are simulations of the experiment using ten randomly oriented pure input states, while dashed lines are simulations using the ten experimental input states (as determined using state tomography, and, in particular, accounting for the decline in their purity as $x$ increases). Squares are experimentally estimated process infidelities. Red and blue represent different depolarization strengths. Inset: "Completeness" of input sets. The inset shows the determinant of the probe states' Gram matrix (normalized to a maximum of 1), whose inverse appears in the tomographic reconstruction. It depends on $x$; larger determinants yield a more robust inversion, while zero determinant indicates a tomographically incomplete set. We observe that a $N 00 N$ fiducial state $(x=1 / 2)$ yields a probe set that is not tomographically complete, while for $x \approx 0.15$, the reconstruction should be as robust and accurate as possible. The main plot confirms this theoretical prediction.

Since theoretical analysis predicts that the $x \approx 0.15$ ensemble should outperform every other $S U(2)$-generated ensemble at QPT (including the nominally more sensitive $N 00 N$ ensemble), we perform an experiment to test the prediction. We prepare several different $S U(2)$-covariant sets of input states-each generated by applying ten different collective polarization $[S U(2)]$ rotations to a single fiducial state with values of $x$ ranging from 0 to 0.47 as detailed in Fig. 1(d) — and use them to perform QPT on an $S U(2)$-jitter process. No a priori assumptions are made about the nature or structure of the process [40]. We perform QPT by (1) preparing many copies of each of the ten states, (2) sending them through the process, (3) performing a tomographically complete set of measurements on each output state, and (4) using maximum-likelihood estimation to reconstruct the process.
Evaluating the performance of an experimental tomographic procedure (i.e., to rank our three different input ensembles) is nontrivial. We cannot assume that we know the "true" process, yet the standard metric of tomographic success is "How close is the reconstructed process to the 'true' process?" We circumvent this problem by using a measure of accuracy that can be estimated directly, the average process infidelity(API) [41]. The API between two processes $\mathcal{E}$ and $\mathcal{F}$ is the (mixed-state) quantum infidelity $1-F(\mathcal{E}[|\psi\rangle\langle\psi|], \mathcal{F}[|\psi\rangle\langle\psi|])$, averaged over all pure inputs to the process according to the unitarily invariant Haar measure:

$$
\operatorname{API}(\mathcal{E}, \mathcal{F})=\int_{\text {Haar }}[1-F(\mathcal{E}[|\psi\rangle\langle\psi|], \mathcal{F}[|\psi\rangle\langle\psi|])] d \psi
$$

The API vanishes for a perfect reconstruction, and increases with errors in tomography.

We estimate the true process using QPT; $\hat{D}_{\gamma}$ is our estimate. To quantify the quality of $\hat{D}_{\gamma}$, we empirically measure the API by 1 . Prepare (many copies of) 40 different randomly chosen input states $\rho_{i}(i=1, \ldots, 40)$. 2. Use state tomography to obtain an estimate $\hat{\rho}_{i}$ of each input state. 3. Apply the depolarization process $\left(D_{\gamma}\right)$ to each state. 4. Use state tomography to obtain an empirical estimate $\hat{\rho}_{i}^{\prime}$ of each output state. 5. Compute the quantum fidelity between (a) the empirical output state $\hat{\rho}_{i}^{\prime}$ and (b) the output state predicted by our QPT estimate, $\hat{D}_{\gamma}\left[\rho_{i}\right]$. 6. Average this fidelity over all 40 input states. The resulting number requires no a priori assertion about the "true" process, and it is a good quantifier of how accurately the QPT estimate $\hat{D}_{\gamma}$ predicts independent experimental results. But it is also an estimate of the theoretical API as defined in Ref. [41], and deviates from it only inasmuch as (i) we have approximated the integral in Eq. (13) by a sum over 40 random states, (ii) those states are not quite pure, and (iii) state tomography on finitely many samples is never quite perfect $(\hat{\rho} \neq \rho)$.

Figure 4 shows the dependence of the empirical API on the fiducial state parameter $x$, for two different depolarization strengths: $\gamma=0.5 \mathrm{rad}$ (blue) and $\gamma=1.5 \mathrm{rad}$ (red). Points represent experimentally measured APIs, while solid and dashed lines represent two different simulations of our experiment. The solid lines are generated by simulating process tomography using ten randomly chosen pure input states; the dashed lines are generated by simulating process tomography using the same ten nearly pure input states used in the experiment. Both simulations use 40 different random states to estimate the empirical API, just as in the experiment.

We observe a minimum in the API (i.e., optimal reconstruction fidelity) at $x \approx 0.15$ for both of the 
depolarization strengths-exactly where theory predicted. This minimum API coincides with the maximum value of $\operatorname{det}(M)$ (Fig. 4, inset). We also confirm that spin-coherent probe ensembles are not optimal. $N 00 N$ state ensembles consistently generate the least accurate QPT. Ironically, while the $N 00 N$ ensemble should in theory fail catastrophically, it is (slightly) redeemed by experimental imperfections in state preparation, which result in the N00N ensemble being not quite perfectly incomplete. However, it still achieves a much worse API than any other $S U(2)$ covariant input ensemble.

\section{CONCLUSIONS}

It is well known that entanglement can (and usually does) improve metrology. However, it has also been taken for granted that ensembles of coherent states (which, in multiphoton systems, are not entangled at all) are "good enough" for process tomography. We show that both of these beliefs should be interpreted cautiously. On one hand, while maximally entangled $N 00 N$ states are indeed optimal for detecting a particularly common and important form of decoherence, they are very bad for characterizing it in detail. And while (spin-)coherent states are indeed sufficient for QPT, they are not optimal. Our results can be summarized as showing that the most robust and flexible way to probe decoherence is with "partly entangled" states, intermediate between $N 00 \mathrm{~N}$ and coherent states.

Our experimental results show that in the presence of prior information [the system is undergoing pure $S U(2)$ jitter], the optimal biphoton probe states are $N 00 N$ states. We expect that this result will be of utility in magnetometry and atomic physics, where interferometry is often used to estimate noise. On the other hand, in the complete absence of prior information, we show that an intermediate entangled state is much better at performing QPT. Our method generalizes the technique of Ref. [11] — preparing a single fiducial state and displacing it - to generate a set of states we believe are optimal for performing QPT on any process, not just $S U(2)$ jitter. This set of states forms a 2-design, and our work is the first experimental evidence confirming that 2-designs are optimal for QPT. Furthermore, we expect the advantage of 2-designs over other sets to increase with the dimensionality of Hilbert space. It is also worth stating that our analysis could be generalized to other symmetry groups, but doing so is an unsolved problem. Our results imply that one can greatly improve the accuracy of QPT by choosing the right set of input states-but, surprisingly, the "right" states for QPT are not those most sensitive to the process. We conclude that detailed state engineering can be very useful in tailoring probe states or ensembles to specific tasks in the characterization (and ultimately remediation) of depolarization.

\section{ACKNOWLEDGMENTS}

We thank Natural Sciences and Engineering Research Council of Canada (NSERC) and Canadian Institute for Advanced Research (CIFAR) for financial support. We also thank Peter Turner and Steven Flammia for helpful discussions. Sandia National Laboratories is a multiprogram laboratory operated by Sandia Corporation, a wholly owned subsidiary of Lockheed Martin Corporation, for the U.S. Department of Energy's National Nuclear Security Administration under Contract No. DE-AC04-94AL85000.

[1] G. Breitenbach, S. Schiller, and J. Mlynek, Measurement of the Quantum States of Squeezed Light, Nature (London) 387, 471 (1997).

[2] A. N. Boto, P. Kok, D. S. Abrams, S. L. Braunstein, C. P. Williams, and J.P. Dowling, Quantum Interferometric Optical Lithography: Exploiting Entanglement to Beat the Diffraction Limit, Phys. Rev. Lett. 85, 2733 (2000).

[3] M. W. Mitchell, J. S. Lundeen, and A. M. Steinberg, Super-resolving Phase Measurements with a Multiphoton Entangled state, Nature (London) 429, 161 (2004).

[4] P. Walther, J.-W. Pan, M. Aspelmeyer, R. Ursin, S. Gasparoni, and A. Zeilinger, de Broglie Wavelength of a Non-Local Four-Photon State, Nature (London) 429, 158 (2004).

[5] V. Giovannetti, S. Lloyd, and L. Maccone, Advances in Quantum Metrology, Nat. Photonics 5, 222 (2011).

[6] P. J. D. Crowley, A. Datta, M. Barbieri, and I. A. Walmsley, Tradeoff in Simultaneous Quantum-Limited Phase and Loss Estimation in Interferometry, Phys. Rev. A 89, 023845 (2014).

[7] P. C. Humphreys, M. Barbieri, A. Datta, and I. A. Walmsley, Quantum Enhanced Multiple Phase Estimation, Phys. Rev. Lett. 111, 070403 (2013).

[8] X.-Q. Zhou, H. Cable, R. Whittaker, P. Shadbolt, J. L. O'Brien, and J.C.F. Matthews, Quantum-Enhanced Tomography of Unitary Processes, arXiv:1402.2897.

[9] M. W. Mitchell, C. W. Ellenor, S. Schneider, and A. M. Steinberg, Diagnosis, Prescription, and Prognosis of a Bell-State Filter by Quantum Process Tomography, Phys. Rev. Lett. 91, 120402 (2003).

[10] J. L. O’Brien, G. J. Pryde, A. Gilchrist, D. F. V. James, N. K. Langford, T. C. Ralph, and A. G. White, Quantum Process Tomography of a Controlled-NOT Gate, Phys. Rev. Lett. 93, 080502 (2004).

[11] M. Lobino, D. Korystov, C. Kupchak, E. Figueroa, B. C. Sanders, and A. I. Lvovsky, Complete Characterization of Quantum-Optical Processes, Science 322, 563 (2008).

[12] R. J. Glauber, Coherent and Incoherent States of the Radiation Field, Phys. Rev. 131, 2766 (1963).

[13] R. Blume-Kohout and P.S. Turner, The Curious Nonexistence of Gaussian 2-Designs, Commun. Math. Phys. 326, 755 (2014).

[14] A. J. Scott, Optimizing Quantum Process Tomography with Unitary 2-Designs, J. Phys. A 41, 055308 (2008). 
[15] A. Fernández-Pérez, A. B. Klimov, and C. Saavedra, Quantum Process Reconstruction Based on Mutually Unbiased Basis, Phys. Rev. A 83, 052332 (2011).

[16] A. Rivas and A. Luis, SU(2)-Invariant Depolarization of Quantum States of Light, Phys. Rev. A 88, 052120 (2013).

[17] D. H. Mahler, L. Rozema, A. Darabi, and A. M. Steinberg, Identification of Decoherence-Free Subspaces without Quantum Process Tomography, Phys. Rev. A 86, 052101 (2012).

[18] L. Viola, E. Knill, and S. Lloyd, Dynamical Generation of Noiseless Quantum Subsystems, Phys. Rev. Lett. 85, 3520 (2000).

[19] L. Viola, E. M. Fortunato, M. A. Pravia, E. Knill, R. Laflamme, and D. G. Cory, Experimental Realization of Noiseless Subsystems for Quantum Information Processing, Science 293, 2059 (2001).

[20] Y. I. Bogdanov, M. V. Chekhova, S. P. Kulik, G. A. Maslennikov, A. A. Zhukov, C. H. Oh, and M. K. Tey, Qutrit State Engineering with Biphotons, Phys. Rev. Lett. 93, 230503 (2004).

[21] R. B. A. Adamson, L. K. Shalm, M. W. Mitchell, and A. M. Steinberg, Multiparticle State Tomography: Hidden Differences, Phys. Rev. Lett. 98, 043601 (2007).

[22] R. B. A. Adamson, P. S. Turner, M. W. Mitchell, and A. M. Steinberg, Detecting Hidden Differences via Permutation Symmetries, Phys. Rev. A 78, 033832 (2008).

[23] F. T. Arecchi, E. Courtens, R. Gilmore, and H. Thomas, Atomic Coherent States in Quantum Optics, Phys. Rev. A 6 , 2211 (1972).

[24] H. F. Hofmann and T. Ono, High-Photon-Number Path Entanglement in the Interference of Spontaneously Down-Converted Photon Pairs with Coherent Laser Light, Phys. Rev. A 76, 031806 (2007).

[25] J. Fiurášek, Conditional Generation of N-Photon Entangled States of Light, Phys. Rev. A 65, 053818 (2002).

[26] I. Afek, O. Ambar, and Y. Silberberg, High-N00N States by Mixing Quantum and Classical Light, Science 328, 879 (2010).

[27] S. Rosen, I. Afek, Y. Israel, O. Ambar, and Y. Silberberg, Sub-Rayleigh Lithography Using High Flux Loss-Resistant Entangled States of Light, Phys. Rev. Lett. 109, 103602 (2012).

[28] Y. Israel, I. Afek, S. Rosen, O. Ambar, and Y. Silberberg, Experimental Tomography of N00N States with Large Photon Numbers, Phys. Rev. A 85, 022115 (2012).
[29] L. A. Rozema, J. D. Bateman, D. H. Mahler, R. Okamoto, A. Feizpour, A. Hayat, and A. M. Steinberg, Scalable Spatial Superresolution Using Entangled Photons, Phys. Rev. Lett. 112, 223602 (2014).

[30] G.S. Agarwal, Relation between Atomic CoherentState Representation, State Multipoles, and Generalized Phase-Space Distributions, Phys. Rev. A 24, 2889 (1981).

[31] L. K. Shalm, R. B. A. Adamson, and A. M. Steinberg, Squeezing and Over-Squeezing of Triphotons, Nature (London) 457, 67 (2009).

[32] M. A. Nielsen and I. L. Chuang, Quantum Computation and Quantum Information, 1st ed. (Cambridge University Press, Cambridge, England, 2000).

[33] H.F Hofmann, Generation of Highly Nonclassical N-Photon Polarization States by Superbunching at a Photon Bottleneck, Phys. Rev. A 70, 023812 (2004).

[34] See Supplemental Material at http://link.aps.org/ supplemental/10.1103/PhysRevX.4.041025 for a derivation of the state-dependent sensitivity to depolarization, and a derivation of the Gram matrices for several different states.

[35] R. Okamoto, H. F. Hofmann, T. Nagata, J. L. O'Brien, K. Sasaki, and S. Takeuchi, Beating the Standard Quantum Limit: Phase Super-Sensitivity of N-Photon Interferometers, New J. Phys. 10, 073033 (2008).

[36] W. K. Wootters and B. D. Fields, Optimal StateDetermination by Mutually Unbiased Measurements., Ann. Phys. (N.Y.) 191, 363 (1989).

[37] J. M. Renes, R. Blume-Kohout, A. J. Scott, and C. M. Caves, Symmetric Informationally Complete Quantum Measurements, J. Math. Phys. (N.Y.) 45, 2171 (2004).

[38] Z. E. D. Medendorp, F. A. Torres-Ruiz, L. K. Shalm, G. N. M. Tabia, C. A. Fuchs, and A. M. Steinberg, Experimental Characterization of Qutrits Using Symmetric Informationally Complete Positive Operator-Valued Measurements, Phys. Rev. A 83, 051801 (2011).

[39] R. B. A. Adamson and A. M. Steinberg, Improving Quantum State Estimation with Mutually Unbiased Bases, Phys. Rev. Lett. 105, 030406 (2010).

[40] We also verify experimentally that our process preserves the biphoton structure-i.e., it does not violate permutation symmetry, and therefore does not change the population in the antisymmetric subspace-and so we can treat it as a quantum process on a three-dimensional Hilbert space.

[41] A. Gilchrist, N. K. Langford, and M. A. Nielsen, Distance Measures to Compare Real and Ideal Quantum Processes, Phys. Rev. A 71, 062310 (2005). 